\title{
Clinicopathological features of breast cancer patients with internal mammary and/or supraclavicular lymph node recurrence without distant metastasis
}

Hitoshi Inari ${ }^{1}$, Natsuki Teruya ${ }^{1}$, Miki Kishi ${ }^{1}$, Rie Horii ${ }^{2,3}$, Futoshi Akiyama ${ }^{2,3}$, Shunji Takahashi ${ }^{4}$, Yoshinori Ito ${ }^{1}$, Takayuki Ueno ${ }^{1 *}$ (D), Takuji Iwase ${ }^{1}$ and Shinji Ohno ${ }^{1}$

\begin{abstract}
Background: Internal mammary and/or supraclavicular (IM-SC) lymph node (LN) recurrence without distant metastasis (DM) in patients with breast cancer is rare, and there have been few reports on its clinical outcomes.

Methods: We enrolled 4237 patients with clinical stage I-IIIC breast cancer treated between January 2007 and December 2012. Clinicopathological features of patients with IM-SC LN recurrence and patients with DM were retrospectively reviewed.
\end{abstract}

Results: With a median follow-up time 78 (range, 13-125) months after the primary operation, 14 (0.3\%) had IM-SC LN recurrence without DM and 274 (6.5\%) had DM at the first recurrence among 4237 patients. No statistical differences were found in the baseline characteristics of the primary tumor between the two groups. The 5-year overall survival (OS) rate after recurrence in patients with IM-SC LN recurrence was 51\% compared with $27 \%$ in patients with DM $(P=0.040)$. In patients with IM-SC LN recurrence, clinically positive axillary LN at diagnosis and pathologically positive axillary LN at primary surgery were poor prognostic factors for distant disease-free survival (DDFS) $(P=0.004$ and 0.007 , respectively). Clinical and pathological axillary nodal status at primary surgery was associated with OS ( $P=0.011$ and 0.001 , respectively).

Conclusions: Patients with IM-SC LN recurrence without DM who had no clinical and pathological axillary LNs involved at primary surgery had a favorable prognosis. A larger validation study is required.

Keywords: Breast cancer, Internal mammary lymph node recurrence, Supraclavicular lymph node recurrence, Prognosis

\section{Background}

The definition of regional lymph node (LN) in breast cancer has been controversial in terms of anatomical extent. Supraclavicular LN metastasis in patients with breast cancer was classified as distant metastasis (DM)

\footnotetext{
* Correspondence: takayuki.ueno@jfcr.or.jp

'Breast Oncology Center, Cancer Institute Hospital, Japanese Foundation for Cancer Research, 3-8-31 Ariake, Koto-ku, Tokyo 135-8550, Japan

Full list of author information is available at the end of the article
}

in the fifth edition of the American Joint Committee on Cancer staging manual for breast cancer, but it has more recently been classified as local disease since the sixth edition $[1,2]$.

Patients with internal mammary and/or supraclavicular (IM-SC) LN recurrence are reported to have better clinical outcome than those with DM. Previous studies have reported that 5-year overall survival (OS) rates after SC LN recurrence and distant recurrence were 33.6 and

(c) The Author(s). 2020 Open Access This article is licensed under a Creative Commons Attribution 4.0 International License, which permits use, sharing, adaptation, distribution and reproduction in any medium or format, as long as you give appropriate credit to the original author(s) and the source, provide a link to the Creative Commons licence, and indicate if changes were made. The images or other third party material in this article are included in the article's Creative Commons licence, unless indicated otherwise in a credit line to the material. If material is not included in the article's Creative Commons licence and your intended use is not permitted by statutory regulation or exceeds the permitted use, you will need to obtain permission directly from the copyright holder. To view a copy of this licence, visit http://creativecommons.org/licenses/by/4.0/ The Creative Commons Public Domain Dedication waiver (http://creativecommons.org/publicdomain/zero/1.0/) applies to the data made available in this article, unless otherwise stated in a credit line to the data. 
9.1\%, respectively [3]. However, patients with IM-SC $\mathrm{LN}$ recurrence have a worse clinical outcome than those with ipsilateral breast tumor recurrence [3-5].

Isolated regional $\mathrm{LN}$ recurrence, except for ipsilateral axillary LN recurrence, is uncommon, with a reported frequency of range 1-5.4\% [6-13]. In particular, IM-SC $\mathrm{LN}$ recurrence without DM is rare; therefore, conducting a prospective randomized trial to compare different treatment strategies is difficult and few retrospective studies have shown long-term outcomes with IM-SC LN recurrence [3]. Thus, the clinical management of IM-SC $\mathrm{LN}$ recurrence without DM in patients with breast cancer is generally empirical, especially in terms of whether cure can be aimed at.

In the present study, we retrospectively reviewed data from patients with primary breast cancer who underwent surgery between 2007 and 2012 and experienced IM-SC LN recurrence and DM during follow-up. We analyzed the clinicopathological characteristics associated with survival after IM-SC LN recurrence in order to uncover groups of patients who have favorable survival outcome and, thus, may benefit from treatment at curative intent.

\section{Methods}

\section{Patients}

Data from patients treated at the Breast Oncology Center, Cancer Institute Hospital, Japanese Foundation for Cancer Research, Tokyo, between January 2007 and
December 2012 were collected. Inclusion criteria included: histologically proven invasive breast cancer, clinical stage I-IIIC, those patients who received surgery from January 2007 to December 2012, and those treated at the Cancer Institute Hospital. Exclusion criteria included: bilateral breast cancer and male. Of these, 706 patients with bilateral breast cancer and seven male patients were excluded, leaving 4237 patients in this study (Fig. 1). Clinicopathological characteristics of the patients are shown in Table S1. We retrospectively reviewed the database and identified patients who experienced IM-SC LN recurrence without DM and those who experienced DM at the first recurrence during the follow-up period. The data of patients in this study are in Additional file 2.

\section{Definition of clinical LN status at diagnosis}

All patients underwent LN evaluation by palpation and ultrasonography prior to primary surgery. Metastasis was confirmed by aspiration cytology [14].

\section{Adjuvant therapy}

Adjuvant therapy was administered based on the guidelines provided by the Japanese Breast Cancer Society [15]. Anthracycline and/or taxane regimens were used depending on risk factors, such as tumor size, nodal status, estrogen receptor (ER) and progesterone receptor (PR) status, human epidermal growth factor receptor 2

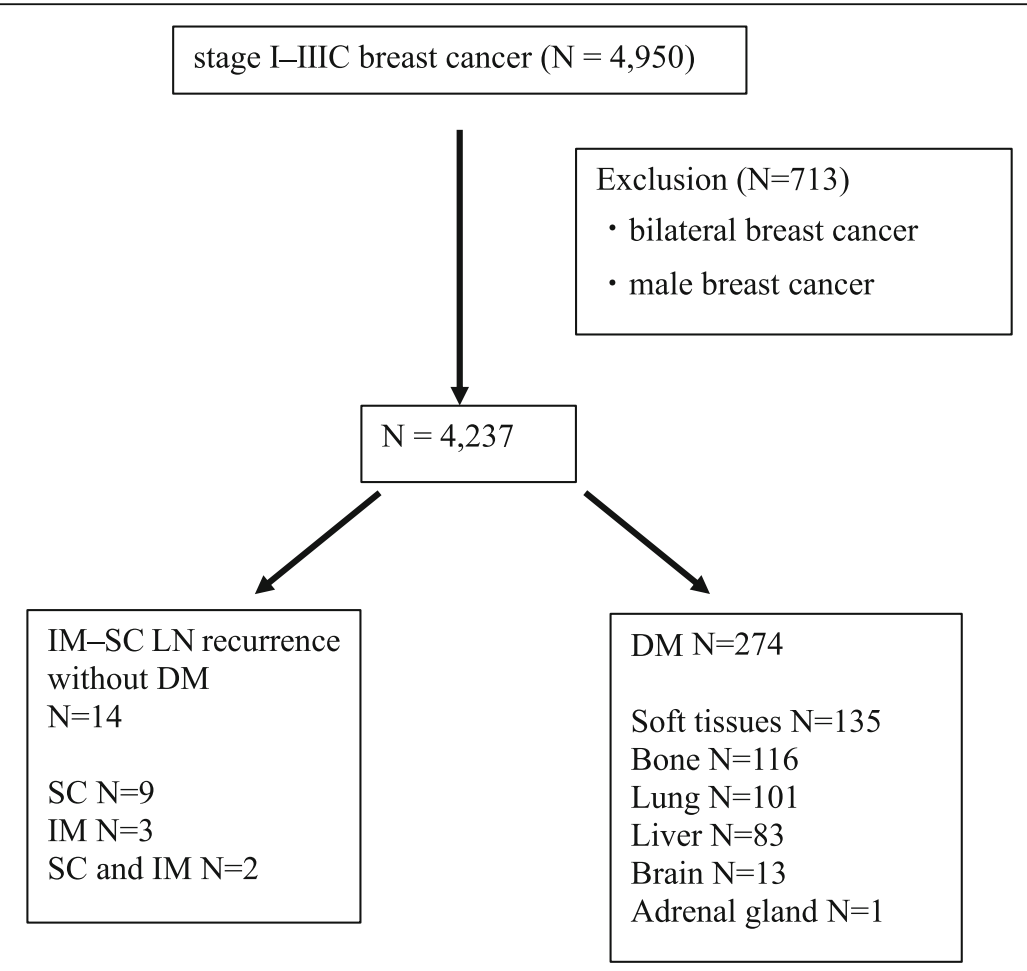

Fig. 1 Flow diagram of the study. DM distant metastasis, IM-SC Internal mammary and/or supraclavicular, LN lymph node, $N$ number 
(HER2) status, nuclear grade, and Ki-67 status. Anthracycline regimens involved 4-6 cycles of adriamycinbased or epirubicin-based regimen as described previously [16]. Taxane regimens included weekly paclitaxel or tri-weekly docetaxel [16]. Endocrine and anti-HER2 therapy was used according to the hormone receptor and/or HER2 status. Post-mastectomy radiotherapy (PMRT) was administered in patients with $\geq 4$ positive nodes, 1-3 positive nodes with extensive lymphatic invasion, IM-SC LN metastasis, or inflammatory breast cancer. PMRT was given in the chest wall and the area of regional LNs. The prescribed dose was 50Gy in 25 fractions of $2 \mathrm{~Gy}$.

\section{Follow-up}

From January 2007 to December 2015, regular postoperative palpation examinations, chest X-ray, and measurements of CEA and CA15-3 were performed every 6 months, and breast ultrasonography and mammography were performed annually. From January 2016 to October 2017 regular postoperative palpation examinations was performed every 6 months up to 5 years, and ultrasonography and mammography were performed annually up to 10 years after operation [14].

\section{Definition of IM-SC LN recurrence}

IM-SC LN recurrence was confirmed by pathological examinations such as aspiration cytology. IM-SC LN recurrence without DM was defined as no evidence of DM at the diagnosis of IM-SC LN recurrence regardless of locoregional recurrence including ipsilateral axillary LN recurrence. A systemic survey after the diagnosis of IMSC LN recurrence included whole-body computed tomography $(\mathrm{CT})$, bone scintigraphy, and positron emission tomography/CT. The area of IM-SC LNs was determined with reference to the irradiation area of radiotherapy [17].

\section{Therapy of IM-SC LN recurrence without DM}

Locoregional radiotherapy was indicated for patients not previously irradiated. Locoregional radiotherapy was given in the area of IM-SC LNs and/or the chest wall or breast. The prescribed dose was 50Gy in 25 fractions of 2 Gy. In addition to local therapy, anthracycline and/or taxane were administered to patients who had not previously received either agent as adjuvant therapy for their primary tumor, as well as endocrine and anti-HER-2 therapy according to the tumor's hormone receptor and HER2 status.

\section{Immunohistochemical analysis}

Immunohistochemical analysis of ER, PR and HER2 expression was performed as described previously [18]. Samples were considered positive for ER and PR if there was a staining of $\geq 10 \%$ of tumor cell nuclei. Expression of HER2 was classified into four groups: $0,1+, 2+$, and $3+$. Samples with $2+$ expression were further tested by in situ hybridization to identify gene amplification. HER2 positivity was defined as HER2 protein 3+ or HER2 gene amplification.

\section{Follow-up data}

Follow-up data until October 31, 2017 were collected using the database. During the study period, no patient was lost to follow-up. We retrospectively reviewed clinicopathological characteristics (including menopausal status, tumor size, LN metastasis, hormone receptor status, and HER2 status), treatment modality (surgery, chemotherapy, endocrine therapy, anti-HER2 therapy and radiotherapy), disease-free interval, IM-SC LN recurrence status (number of metastatic LNs and number of areas of metastatic LNs), and distant disease-free survival (DDFS) and OS. Pathological TNM classification was based on the Union for International Cancer Control staging system (eighth edition) [19]. DDFS was defined as the period from the day of diagnosis of locoregional recurrence until the day of diagnosis of distant metastasis or death from any cause. OS after recurrence was defined as the period from the day of diagnosis of breast cancer recurrence until the day of death from any cause. Median follow-up time was 78 (range, 13-125) months after the primary operation and 22 (range, $1-85$ ) months after the recurrence.

We obtained informed consent from all patients, and the Ethics Committees of the institute approved the study protocol (\# 2018-1100).

\section{Statistical analysis}

Clinicopathological characteristics were compared by ttests and chi-square tests. The Kaplan-Meier method was used to determine DDFS and OS, and survival curves were compared using the log-rank test. All $P$ values were two tailed, and $P<0.05$ was considered statistically significant. Statistical analysis was performed using IBM SPSS statistics 20 (SPSS Inc., Chicago, IL, USA).

\section{Results}

Clinicopathological features of patients with IM-SC LN recurrence without DM and those with DM

Among 4237 patients with breast cancer whose background characteristics are shown in Table S1, 14 (0.3\%) had IM-SC LN recurrence without DM and 274 (6.5\%) had DM (Fig. 1, Table 1). The median time to recurrence was 30 months in patients with IM-SC LN recurrence and 33 months in those with DM. The number of patients with different recurrence sites was shown in Fig. 1. No statistical differences were found in the baseline characteristics of the primary tumor between the two groups (Table 1). The summary of the initial treatment 
Table 1 Clinicopathological characteristics of patients with IM-SC LN recurrence and DM

\begin{tabular}{|c|c|c|c|}
\hline Characteristics & Patients with IM-SC LN recurrence $(n=14)$ & Patients with distant metastasis $(n=274)$ & $P$-value \\
\hline Disease-free survival, month (mean \pm SD) & $30.71 \pm 6.87$ & $33.25 \pm 1.63$ & 0.732 \\
\hline Age, years (mean $\pm S D)$ & $45.36 \pm 3.892$ & $52.5 \pm 12.93$ & 0.093 \\
\hline \multicolumn{4}{|l|}{ Menopausal status at primary surgery } \\
\hline Pre- & 9 & 133 & \multirow[t]{2}{*}{0.250} \\
\hline Post- & 5 & 141 & \\
\hline \multicolumn{4}{|l|}{ Clinical T stage $e^{a}$} \\
\hline T1 & 3 & 44 & \multirow[t]{4}{*}{0.615} \\
\hline $\mathrm{T} 2$ & 9 & 160 & \\
\hline T3 & 2 & 41 & \\
\hline T4 & 0 & 29 & \\
\hline \multicolumn{4}{|l|}{ Clinical N stage $\mathrm{a}^{\mathrm{a}}$} \\
\hline 0 & 6 & 121 & \multirow[t]{4}{*}{0.732} \\
\hline 1 & 6 & 102 & \\
\hline 2 & 1 & 9 & \\
\hline 3 & 1 & 42 & \\
\hline \multicolumn{4}{|l|}{ Clinical stage $e^{a}$} \\
\hline । & 2 & 32 & \multirow[t]{3}{*}{0.311} \\
\hline$\|$ & 10 & 149 & \\
\hline$\|$ & 2 & 93 & \\
\hline \multicolumn{4}{|l|}{ Perioperative chemotherapy } \\
\hline No & 4 & 52 & \multirow[t]{2}{*}{0.376} \\
\hline Yes & 10 & 222 & \\
\hline \multicolumn{4}{|l|}{ Surgical procedure of the primary tumor } \\
\hline Partial mastectomy & 4 & 79 & \multirow[t]{2}{*}{0.983} \\
\hline Mastectomy & 10 & 195 & \\
\hline \multicolumn{4}{|l|}{ Pathological LN status of the primary tumor } \\
\hline Negative & 7 & 87 & \multirow[t]{2}{*}{0.156} \\
\hline Positive & 7 & 187 & \\
\hline \multicolumn{4}{|l|}{ LI status of the primary tumor } \\
\hline Negative & 6 & 87 & \multirow[t]{2}{*}{0.260} \\
\hline Positive & 8 & 187 & \\
\hline \multicolumn{4}{|l|}{ ER status } \\
\hline Positive & 5 & 168 & \multirow[t]{2}{*}{0.152} \\
\hline Negative & 9 & 105 & \\
\hline \multicolumn{4}{|l|}{ HER2 status } \\
\hline Positive & 5 & 35 & \multirow[t]{2}{*}{0.111} \\
\hline Negative & 9 & 238 & \\
\hline
\end{tabular}

aTM classification is shown based on the eighth edition of the Union for International Cancer Control staging system $D M$ Distant metastasis, ER, Estrogen receptor, HER2 Human epidermal growth factor receptor 2, IM-SC Internal mammary and/or supraclavicular, LN Lymph node, LI Lymphatic invasion, SD Standard deviation

after IM-SC LN recurrence without DM is shown in Table S3 and S4. Surgery was performed in two patients. They underwent removal of swollen LNs in IM or SC regions for the purpose of biopsy to confirm breast cancer metastasis. No dissection of IM or SC regions was performed. One patient with IM-SC LN recurrence refused any treatment.

We examined OS after recurrence in patients with IM-SC LN recurrence without DM and those with DM (Fig. 2). The median follow-up after recurrence was 22 


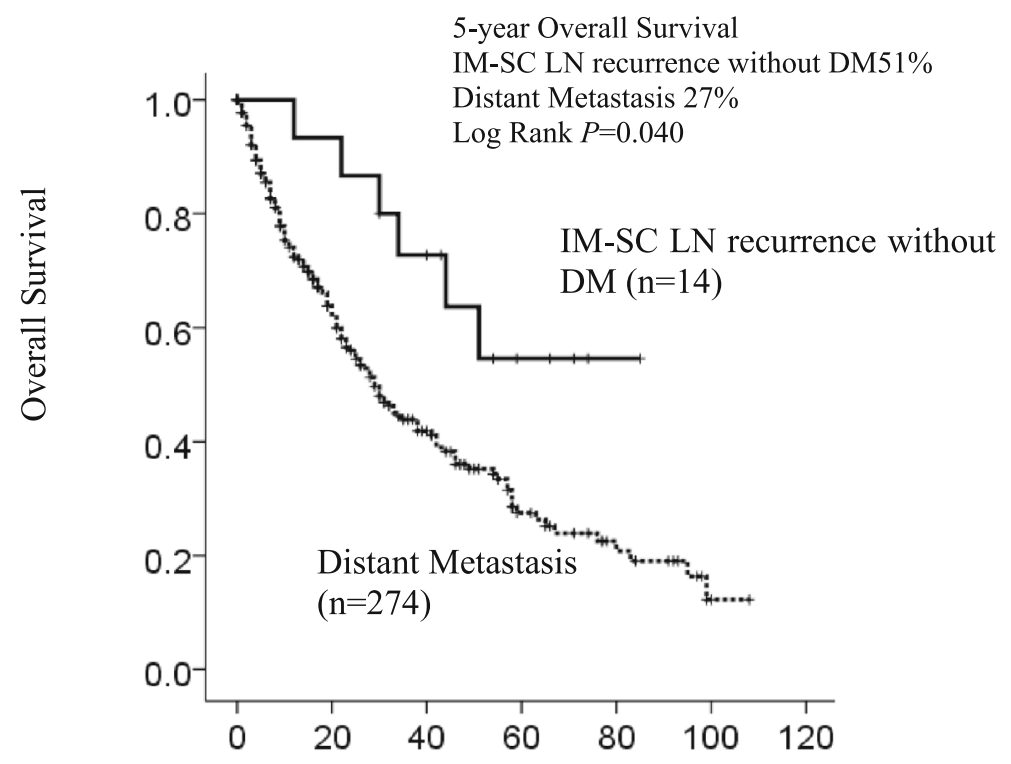

No. at Risk Months after Recurrence

$\begin{array}{ccccccc}\text { IM-SC LN } & 14 & 10 & 8 & 3 & 1 & 0 \\ \text { Distant } & 274 & 254 & 234 & 25 & 12 & 1\end{array}$

Fig. 2 Survival Outcomes between patients with IM-SC LN recurrence without DM and those with DM. Kaplan-Meier curve for overall survival after recurrence in patients with IM-SC LN recurrence without DM $(n=14)$ and patients with DM $(n=274)$. The 5 -year OS rate in patients with IM-SC LN recurrence without DM was 51\% compared with 27\% in patients with DM recurrence $(P=0.040)$. DM distant metastasis, IM-SC LN internal mammary and/or supraclavicular lymph node, OS overall survival

months. The 5-year OS rate in patients with IM-SC LN recurrence was $51 \%$ compared with $27 \%$ in patients with DM. Patients with IM-SC LN recurrence had significantly better OS than patients with DM $(P=0.040)$.

\section{Clinicopathological factors associated with DDFS and OS in patients with IM-SC LN recurrence}

We examined factors associated with DDFS after recurrence in patients with IM-SC LN recurrence. Prognostic factors associated with DDFS were clinical axillary LN status at diagnosis, pathological axillary LN status of the primary tumor, and PMRT (Table 2). The log-rank test showed significantly better DDFS in patients with clinical axillary node-negative tumors at diagnosis $(P=$ 0.004, Fig. 3a), and OS ( $P=0.011$, Fig. 3b). Patients with pathological axillary node-negative tumor at the primary surgery showed better DDFS than those with axillary node-positive tumor $(P=0.007$, Fig. 3c). The 5-year DDFS rate was $0 \%$ in patients with pathological axillary node-positive tumor at the primary surgery while it was $69 \%$ in those with axillary node-negative tumor. Similarly, patients with pathological axillary node-negative tumor at the primary surgery had better OS $(P=0.001$, Fig. 3d). The 5 -year OS rate was $100 \%$ in patients with pathological axillary node-negative tumor and $0 \%$ in patients with axillary node-positive tumor.
Because breast cancer subtypes and treatments can affect prognosis of the patients, subtypes and treatments were reviewed according to pathological LN status at primary tumor (Table S3 and S4). Surgery and chemotherapy including trastuzumab use after recurrence were different between the groups, the analysis adjusted by these confounding factors was performed as an exploratory analysis (Table S5). LN status at primary tumor remained an independent factor for DDFS after adjusting by ER, HER2, surgery and chemotherapy $(P=0.03)$ (Table $S 4)$.

\section{Discussion}

The rate of IM-SC LN recurrence without DM was $0.3 \%$ in this study, which is concordant with other reports. The rates of isolated SC LN recurrence and IM LN recurrence were reported to be $0.4-2$ and $0.08 \%$, respectively $[3,6,20,21]$. Because isolated IM-SC LN recurrence is very rare, it is clinically important to accumulate clinical data from different institutions to clarify an optimal treatment strategy for such rare disease.

We confirmed that patients with IM-SC LN recurrence without DM had significantly better OS after recurrence than patients with DM in agreement with the previous reports $[3,6]$. We found that patients with IMSC LN recurrence without DM had good prognosis if axillary LN was negative at the clinical diagnosis and 
Table 2 Univariate analysis of prognostic factors related to DDFS in patients with internal IM-SC LN recurrence

\begin{tabular}{l} 
Prognostic factor \\
\hline Tumor size of primary tumor ${ }^{\mathrm{a}}$ \\
T1 and T2 \\
T3 and T4 \\
Clinical LN status at diagnosis \\
Negative \\
Positive \\
Pathological LN status of primary tumor \\
Negative \\
Positive \\
Primary ER status \\
Negative \\
Positive \\
Primary HER2 status \\
Negative \\
Positive \\
Type of surgery \\
Mastectomy \\
Partial mastectomy \\
Perioperative Chemotherapy \\
No
\end{tabular}

Post mastectomy radiation therapy

Yes

No

Disease free interval

$$
\leq 1 \text { year }
$$

$>1$ year

Number of metastatic lymph nodes at recurrence

$\leq 2$ lymph nodes

$\geq 3$ lymph nodes

8

6

Number of regions of metastatic lymph nodes at recurrence

\section{Patients \\ $(n=14)$}

12

6

7

5

9

9

5

10

10

\section{Univariate analysis}

HR

2.455

11.43

6.637

1.031

1.063

0.570

1.724

4

10

4

10

5.435

0.396
$95 \% \mathrm{Cl}$

$0.472-12.778$

$1.402-93.175$

$\underline{0.023}$

0.964

$0.271-3.929$

$0.667-1.695$

1.063

0.570

0.499

$0.356-8.355$

$1.202-24.581$

$0.95-1.638$

0.201

0.675

$0.168-2.714$

0.580

1

12

1.123

$0.137-9.231$

0.914

Hormone therapy after recurrence

Yes

1.084

$0.257-4.567$

0.913

No

10

Chemotherapy after recurrence

Yes

No

5

Operation after recurrence

Yes

No

12 
Table 2 Univariate analysis of prognostic factors related to DDFS in patients with internal IM-SC LN recurrence (Continued)

\begin{tabular}{lllcr}
\hline Prognostic factor & $\begin{array}{l}\text { Patients } \\
(\boldsymbol{n}=\mathbf{1 4})\end{array}$ & \multicolumn{2}{l}{ Univariate analysis } & \\
\cline { 3 - 4 } & 8 & HR & $\mathbf{9 5 \%} \mathrm{Cl}$ & $0.238-3.408$ \\
\hline Yes & 6 & & \\
No & 6 & & \\
\hline
\end{tabular}

${ }^{\mathrm{a}}$ TNM classification is shown based on the eighth edition of the Union for International Cancer Control staging system

Under bar indicates values that are statistically significant $(P<0.05)$

CI Confidence interval, DDFS Distant disease-free survival, ER Estrogen receptor, HER2 Human epidermal growth factor receptor 2, IM-SC Internal mammary and/or supraclavicular, LN Lymph node

primary surgery. Therefore, the present study suggests that some patients with IM-SC LN recurrence without DM have a favorable prognosis, particularly if axillary LNs are not involved at the clinical diagnosis and primary surgery, thus, it might be possible to consider curative treatment for patients with IM-SC LN recurrence without DM. There are several studies that examined prognostic factors after SC recurrence. Using the Danish Breast Cancer Cooperative Group treatment database, 305 patients with SC LN recurrence with or without other

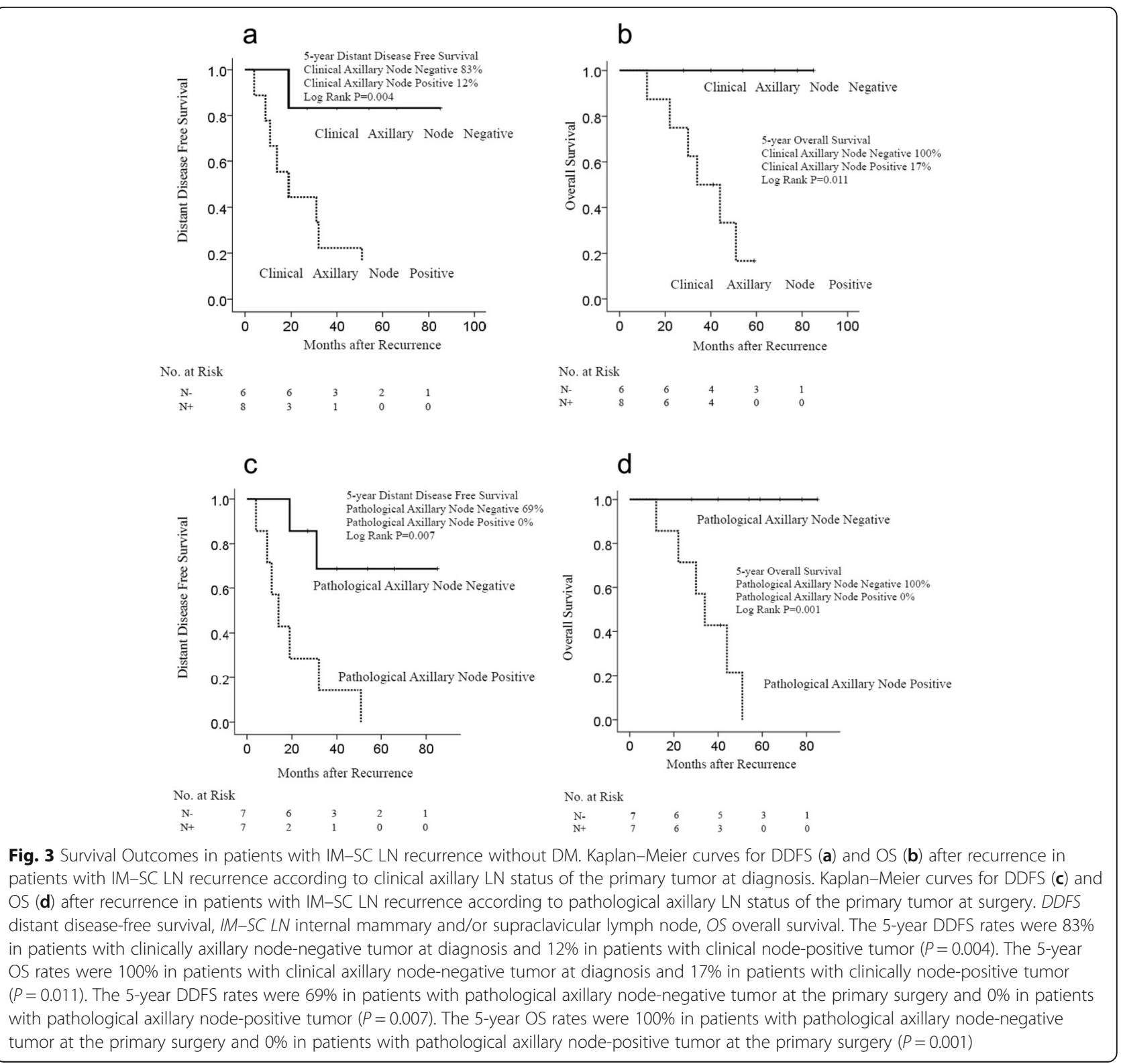


locoregional recurrence were identified [20]. The study showed that the combination of local and systemic treatment, negative nodal status and low grade at primary diagnosis were related to longer progression free survival after SC LN recurrence but that nodal status at primary diagnosis was not associated with OS after recurrence [20]. Another study included 42 patients with SC LN recurrence and found no association between nodal status of primary tumor and DDFS [21]. The discrepancies between studies seem to derive from the differences in perioperative systemic treatment for primary tumor and treatment strategies after regional recurrence. Indeed, these reports were based on the data from patients whose primary tumors were treated between 1977 and 2003 and between 1984 and 1994, respectively [20, 21]. In our study, the chemotherapy regimens for perioperative treatment included anthracycline and taxane and were essentially identical to the current regimens, which, we believe, makes the results more practically useful.

One of the possible explanations for poor prognosis in patients with an axillary node -positive tumor at the primary surgery is the use of adjuvant chemotherapy and PMRT at the time of primary surgery. Possibly, recurrent tumors in those with axillary node-positive disease had acquired resistance to chemotherapy and, in part, radiotherapy. On the contrary, those patients who had no axillary LN involvement of the primary tumor could receive anthracycline or taxane, or both, and radiotherapy after recurrence, which would have, to some extent, resulted in favorable prognosis. Although it is exploratory and needs cautious interpretation with this small sample size, the pathological nodal status remained prognostic for DDFS after adjusting by subtype, chemotherapy and radiotherapy (Table S5).

Our treatment strategy was consistent with the fourth ESO-ESMO International Consensus Guidelines for Advanced Breast Cancer, which recommends the use of systemic therapy (chemotherapy, endocrine therapy, and/or anti-HER2 therapy) for patients with regional recurrence, in addition to local therapy [22]. Locoregional radiotherapy was indicated for patients not previously irradiated. Previous studies showed that local and systemic combination therapy after recurrence was an independent factor for improved outcome and that patients with isolated IM LN recurrence exhibited excellent outcomes when managed with aggressive salvage treatments consisting of chemotherapy, radiation therapy and surgery [20,23].

Curability has been uncertain; thus, the treatment strategy for IM-SC LN recurrence without DM is currently on an individual basis, either palliative or curative. Our results suggest an option for treatment strategy based on axillary nodal status at the primary surgery. Patients with IM-SC LN recurrence without axillary nodal involvement at the primary surgery may receive intensive treatment with curative intent, whereas those with axillary nodal involvement may receive palliative treatment. To confirm the clinical validity of this treatment strategy, a larger study is required.

One of the major limitations in the present study was a small number of patients, which resulted from the rarity of isolated IM-SC LN recurrence. The survival analysis of this small patient population needs to be interpreted with caution. Although we tried to validate our results using external data sources such as SEER database, the information on first recurrence sites including IM-SC LN was missing and so such analyses could not be performed [24]. More patients are necessary to confirm our results; therefore, we are planning a multicenter study focusing on prognosis of isolated regional recurrence. Another limitation was the short follow-up period. It is important to follow the patients for a longer period.

\section{Conclusion}

We found that outcomes in patients with IM-SC LN recurrence without DM who had no axillary nodal involvement at the clinical diagnosis and primary surgery were favorable after recurrence. Therefore, the results of the present study suggest that some patients with IM-SC LN recurrence without DM can consider treatment aiming at cure if they have an axillary node-negative primary tumor.

\section{Supplementary information}

Supplementary information accompanies this paper at https://doi.org/10. 1186/s12885-020-07442-8.

Additional file 1: Table S1 Clinicopathological characteristics of patients with breast cancer. Table S3 Summary of initial treatment after IM-SC LN recurrence. Table S4 Subtypes and treatment of patients with IM-SC LN recurrence without DM according to pathological LN status at primary tumor. Table S5 Multivariate analysis of prognostic factors related to DDFS in patients with IM-SC LN recurrence without DM.

Additional file 2.

\section{Abbreviations \\ CT: Computed tomography; DDFS: Distant disease-free survival; DM: Distant metastasis; ER: Estrogen receptor; HER2: Human epidermal growth factor receptor 2; IM-SC: Internal mammary and/or supraclavicular; LN: Lymph node; OS: Overall survival; PMRT: Post-mastectomy radiotherapy; PR: Progesterone receptor}

Acknowledgments

We thank Ms. Rie Gokan for data management of the Breast Oncology Center.

Authors' contributions

HI, NT, MK, TI and TU designed the study. HI and TU performed clinical, and, statistical investigation, and drafted the manuscript. $\mathrm{RH}$ and FA participated in the histological and immunohistochemical evaluation. ST, YI and SO participated in preparing and drafting the manuscript. The authors read and approved the final manuscript. 


\section{Funding}

This research did not receive any specific grant from funding agencies in the public, commercial, or not-for-profit sectors.

\section{Availability of data and materials}

The dataset supporting the conclusions of this article is included within the article (and its Additional file 2).

\section{Ethics approval and consent to participate}

The Ethics Committees of the Cancer Institute Hospital approved the study protocol (\# 2018-1100). All patients gave consent in writing to participate in this study.

\section{Consent for publication}

Not applicable.

\section{Competing interests}

The authors declare that they have no competing interests.

\section{Author details}

'Breast Oncology Center, Cancer Institute Hospital, Japanese Foundation for Cancer Research, 3-8-31 Ariake, Koto-ku, Tokyo 135-8550, Japan. ${ }^{2}$ Department of Pathology, Cancer Institute Hospital, Japanese Foundation for Cancer Research, 3-8-31 Ariake, Koto-ku, Tokyo 135-8550, Japan. ${ }^{3}$ Department of Pathology, Cancer Institute, Japanese Foundation for Cancer Research, 3-8-31 Ariake, Koto-ku, Tokyo 135-8550, Japan. ${ }^{4}$ Department of Medical Oncology, Cancer Institute Hospital, Japanese Foundation for Cancer Research, 3-8-31 Ariake, Koto-ku, Tokyo 135-8550, Japan.

Received: 9 June 2019 Accepted: 21 September 2020 Published online: 29 September 2020

\section{References}

1. Fleming ID. AJCC cancer staging manual. 5th ed. New York: LippincottRaven; 1997. p. 171-80.

2. Singletary SE, Connolly JL. Breast cancer staging: working with the sixth edition of the AJCC cancer staging manual. CA Cancer J Clin. 2006;56(1):3747quiz 50-1. https://doi.org/10.3322/canjclin.56.1.37.

3. Chen SC, Chang HK, Lin YC, Leung WM, Tsai CS, Cheung YC, et al. Prognosis of breast cancer after supraclavicular lymph node metastasis: not a distant metastasis. Ann Surg Oncol. 2006;13(11):1457-65 Epub 2006 Sep 8.

4. Wapnir IL, Anderson SJ, Mamounas EP, Geyer CE Jr, Jeong JH, Tan-Chiu E, et al. Prognosis after ipsilateral breast tumor recurrence and locoregional recurrences in five National Surgical Adjuvant Breast and bowel project node-positive adjuvant breast cancer trials. J Clin Oncol. 2006;24(13):202837.

5. Anderson SJ, Wapnir I, Dignam JJ, Fisher B, Mamounas EP, Jeong JH, et al. Prognosis after ipsilateral breast tumor recurrence and locoregional recurrences in patients treated by breast-conserving therapy in five National Surgical Adjuvant Breast and bowel project protocols of node-negative breast cancer. J Clin Oncol. 2009;27(15):2466-73. https://doi.org/10.1200/ JCO.2008.19.8424 Epub 2009 Apr 6.

6. Harris EE, Hwang WT, Seyednejad F, Solin LJ. Prognosis after regional lymph node recurrence in patients with stage I-\| breast carcinoma treated with breast conservation therapy. Cancer. 2003;98(10):2144-51.

7. Moran MS, Haffty BG. Local-regional breast cancer recurrence: prognostic groups based on patterns of failure. Breast J. 2002;8(2):81-7.

8. Fisher B, Anderson S, Bryant J, Margolese RG, Deutssh M, Fisher ER, et al. Twenty-year follow-up of a randomized trial comparing total mastectomy, lumpectomy, and lumpectomy plus irradiation for the treatment of invasive breast cancer. N Engl J Med. 2002;347(16):1233-41. https://doi.org/10.1056/ NEJMoa022152.

9. Lukens JN, Vapiwala N, Hwang WT, Solin LJ. Regional nodal recurrence after breast conservation treatment with radiotherapy for women with earlystage breast carcinoma. Int J Radiat Oncol Biol Phys. 2009;73(5):1475-81. https://doi.org/10.1016/j.jjrobp.2008.06.1955.

10. Newman LA, Hunt KK, Buchholz T, Kuerer HM, Vlastos G, Mirza N, et al. Presentation, management and outcome of axillary recurrence from breast cancer. Am J Surg. 2000:180(4):252-6. https://doi.org/10.1016/s00029610(00)00456-6.
11. Konkin DE, Tyldesley S, Kennecke H, Speers CH, Olivotto IA, Noelle D. Management and outcomes of isolated axillary node recurrence in breast cancer. Arch Surg. 2006;141(9):867-72discussion 872-4. https://doi.org/10. 1001/archsurg.141.9.867.

12. Louis-Sylvestre C, Clough K, Asselain B, Vilcoq JR, Salmon RJ, Campana F, et al. Axillary treatment in conservative management of operable breast cancer: dissection or radiotherapy? Results of a randomized study with 15 years of follow-up. J Clin Oncol. 2004;22(1):97-101. https://doi.org/10.1200/ JCO.2004.12.108.

13. Recht A, Gray R, Davidson NE, Fowble BL, Solin LJ, Cummings FJ, et al. Locoregional failure 10 years after mastectomy and adjuvant chemotherapy with or without tamoxifen without irradiation: experience of the eastern cooperative oncology group. J Clin Oncol. 1999;17:1689-700. https://doi. org/10.1200/JCO.1999.17.6.1689.

14. Ogiva A, Kimura K, Nakashima E, Sakai T, Miyagi Y, lijima K, et al. Long-term prognoses and outcomes of axillary lymph node recurrence in 2,578 sentinel lymph node-negative patients for whom axillary lymph node dissection was omitted: results from one Japanese hospital. Breast Cancer. 2016;23(2):318-22. https://doi.org/10.1007/s12282-014-0576-5 Epub 2014 Nov 7.

15. The Japanese Breast Cancer Society Clinical Practice Guidelines for Breast Cancer 2018 http://jbcs.gr.jp/guidline/2018/index/ [Accessed 4 Jan 2019].

16. Fukuda T, Horii R, Gomi N, Miyagi Y, Takahashi S, Ito Y, et al. Accuracy of magnetic resonance imaging for predicting pathological complete response of breast cancer after neoadjuvant chemotherapy: association with breast cancer subtype. Springerplus. 2016;5:152. https://doi.org/10.1186/s40064016-1800-x eCollection 2016.

17. Offersen BV, Boersma L, Kirkove C, Kirkove C, Hol S, Aznar MC, et al. ESTRO consensus guideline on target volume delineation for elective radiation therapy of early stage breast cancer. Radiother Oncol. 2015;114(1):3-10. https://doi.org/10.1016/j.radonc.2014.11.030 Epub 2015 Jan 24.

18. Inoue $H$, Horii R, Ito $Y$, Iwase T, Ohno S, Akiyama F. Tumor-infiltrating lymphocytes affect the efficacy of trastuzumab-based treatment in human epidermal growth factor receptor 2-positive breast cancer. Breast Cancer. 2018;25(3):268-74. https://doi.org/10.1007/s12282-017-0822-8 Epub 2017 Nov 28

19. Brierley JD, Gospodarowicz MK, Wittekind C. TNM classification of malignant tumours. 8th ed. New York: Wiley; 2017.

20. Pedersen AN, Møller S, Steffensen KD, Haahr V, Jensen M, Kempel MM, et al. Supraclavicular recurrence after early breast cancer: a curable condition? Breast Cancer Res Treat. 2011;125(3):815-22. https://doi.org/10.1007/s10549010-0918-8.

21. van der Sangen MJ, Coebergh JW, Roumen RM, Rutten HJ, Vreugdenhil G, Voogd AC. Detection, treatment, and outcome of isolated supraclavicular recurrence in 42 patients with invasive breast carcinoma. Cancer. 2003;98(1): 11-7. https://doi.org/10.1002/cncr.11469.

22. Cardoso F, Senkus E, Costa A, Papadopoulos E, Aapro M, Andre F, et al. 4th ESO-ESMO international consensus guidelines for advanced breast cancer (ABC 4). Ann Oncol. 2018;29:1634-57. https://doi.org/10.1093/annonc/ mdy192 Published online 19 July 2018.

23. Xu AJ, DeSelm CJ, Ho AY, Gillespie EF, Braunstein LZ, Khan AJ, et al. Overall survival of breast cancer patients with Locoregional failures involving internal mammary nodes. Adv Radiat Oncol. 2019;4(3):447-52. https://doi. org/10.1016/j.adro.2019.02.004 eCollection 2019 Jul-Sep.

24. National Cancer Institute Surveillance, Epidemiology, and End Results Program https://seer.cancer.gov/ [Accessed 19 June 2020].

\section{Publisher's Note}

Springer Nature remains neutral with regard to jurisdictional claims in published maps and institutional affiliations. 